\title{
Integrated sensors for PZT actuators based on thick-film resistors
}

\author{
Bergander-A Maeder-T Valencia-B Breguet-JM Ryser-P, "Integrated \\ sensors for PZT actuators based on thick-film resistors", Proceedings, \\ International Symposium on Mechatronics and Human Sciences, Nagoya \\ (JP), 181-186, 2002.
}

C2002 IEEE. Personal use of this material is permitted. However, permission to reprint/republish this material for advertising or promotional purposes or for creating new collective works for resale or redistribution to servers or lists, or to reuse any copyrighted component of this work in other works must be obtained from the IEEE.

\begin{abstract}
A common problem when using piezoceramic actuators for precise positioning is their hysteresis. Either external sensors such as capacitive or inductive probes, optical sensors or strain gauges are commonly used. The latter are the most favorable solution in many cases, as they occupy the smallest volume and disturbances can mostly be avoided. Drawbacks are the assembly of these strain gauges on the target, their small gauge factor and their size. We propose a sensor based on thick-film technology which permits to avoid these problems. It will allow to increase the integration level of subminiature mechatronic systems. A piezoceramic element is covered with an electrode on either side. Afterwards, an insulating layer is patterned, followed by the required number of piezoresistive elements and the necessary electrode pads and conductive tracks. Experiments in the laboratory have shown that position control using these resistances is possible, taking advantage of the reliability, the intimate contact between sensor and actuator, a negligible creep and the stability. In this paper we present several possible sensor configurations, applications and measurement results.
\end{abstract}




\title{
Integrated Sensors for PZT Actuators Based on Thick-Film Resistors
}

\author{
A.Bergander*, T.Maeder**, B. Valencia*, J.-M. Breguet*, and P.Ryser** \\ Swiss Federal Institute of Technology Lausanne (EPFL) \\ *Laboratoire de systèmes robotiques (IPR-LSRO),**Laboratoire de production microtechnique (IPR-LPM) \\ ME 3, Chemin des Machines, CH-1015 Lausanne, Switzerland \\ e-mail: a.bergander@ieee.org, thomas.maeder@epfl.ch
}

\begin{abstract}
A common problem when using piezoceramic actuators for precise positioning is their hysteresis. Either external sensors such as capacitive or inductive probes, optical sensors or strain gauges are commonly used. The latter are the most favorable solution in many cases, as they occupy the smallest volume and disturbances can mostly be avoided. Drawbacks are the assembly of these strain gauges on the target, their small gauge factor and their size.

We propose a sensor based on thick-film technology which permits to avoid these problems. It will allow to increase the integration level of subminiature mechatronic systems. A piezoceramic element is covered with an electrode on either side. Afterwards, an insulating layer is patterned, followed by the required number of piezoresistive elements and the necessary electrode pads and conductive tracks. Experiments in the laboratory have shown that position control using these resistances is possible, taking advantage of the reliability, the intimate contact between sensor and actuator, a negligible creep and the stability. In this paper we present several possible sensor configurations, applications and measurement results.
\end{abstract}

\section{INTRODUCTION}

$\mathbf{I}^{2}$ $\mathrm{N}$ recent years, PZT ceramics have become very popular for positioning applications besides their applications in vibration control and in ultrasonic motors. Today, many companies offer a broad range of positioners based on piezoelectric ceramics for applications such as micropositioning and -assembly, fiber optics, beam deflection or positioning of optical elements.

The strain, which can be obtained by applying an electric field to piezoceramic elements is usually in the order of $0.1 \%$. Therefore the movement is usually amplified. This amplification can be accomplished in different ways [1], such as:

Piezoceramic stacks are generally employed if the available space is restricted and a comparatively large force needs to be generated. They are relatively costly because of the complicated manufactur- ing procedures needed to obtain the multilayer structure.

Levers are often used in combination with the stacked actuators to further increase the range if displacements of several $100 \mu \mathrm{m}$ are needed.

Bimorph or monomorph actuators have the advantage of being very simple and nevertheless offering a large range of movement. The main drawback is their low mechanical stiffness, which results in comparatively low resonance frequencies. Bimorph actuators are nevertheless very interesting for applications where a large displacement needs to be generated by comparatively small elements, and where the generated force has to be in or below the $\mathrm{mN}$ range.

As the hysteresis in soft piezoceramic materials is in the order of $10 \%$ of their displacement range, a position feedback is needed for positioning applications. Optical sensors, as well as capacitive sensors or strain gauges are used to measure the displacement [2], [3]. Strain gauges offer the particular advantage of being inexpensive and relatively simple to mount mechanically and electrically.

Conventional strain gauges, as commercially available from many suppliers, consist usually of thin metal film on an insulating polyimide or epoxy support [4]. The gauge factor $K_{g f}$ for strain gauges is defined with

$$
K_{g f}=\frac{\frac{\Delta R}{R}}{\frac{\Delta l}{l}}
$$

As the piezoresistive effect in metals is very low, $K_{g f}$ depends essentially on the Poisson ratio of the metal $\nu_{m}$ :

$$
K_{g f}=1+2 \nu_{m}
$$

In addition, because of the low resistivity of metals, the foil is usually patterned to a meander-like 
shape by lithography technique. The sensors are applied to the substrate using special glue, which has to match the mechanical properties of the insulating support and which adheres well to the substrate. The strain gauge may afterwards be covered by an elastic protective polymer or epoxy film. Strain gauge setups usually employ the Wheatstone bridge as primary sensing circuit with the strain gauge representing one or more arms of the bridge. A stable DC amplifier with a high gain and offset compensation is used to amplify the bridge output for further processing.

Despite their advantages mentioned above, strain gauges still have a certain number of drawbacks. During operation it may happen that the connection between the substrate and the strain gauge detaches partially due to fatigue. This may decrease the sensitivity of the setup or even cause a nonlinear behavior.

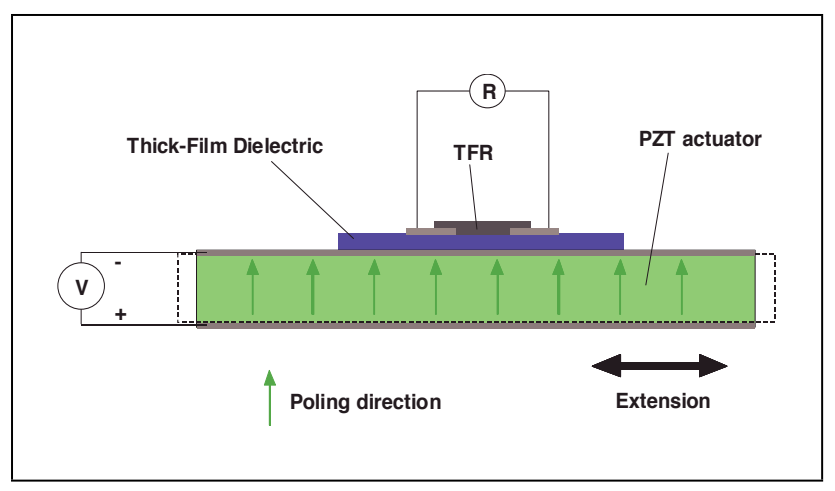

Fig. 1. Transversal actuator with integrated TFR structure

Another inconvenience is the necessity to assemble, which does not allow for cost-effective fabrication of large quantities and the low gauge factor along with the resulting encombrance, as a certain minimum surface for the sensor is needed.

Considering these problems it seems to be advantageous to search for a different way of integrating strain gauges into PZT positioning systems. We propose therefore the direct integration of thick-film resistors onto the piezoelectric ceramics. This allows for an arrangement of resistors for the measurement and temperature compensation and eventually the integration of the whole bridge within a few square millimeters.

\section{THICK-FILM RESISTORS (TFR) IN SENSOR APPLICATIONS}

Thick-film resistors consist of conducting oxide nanoparticles embedded within a glassy matrix [5].

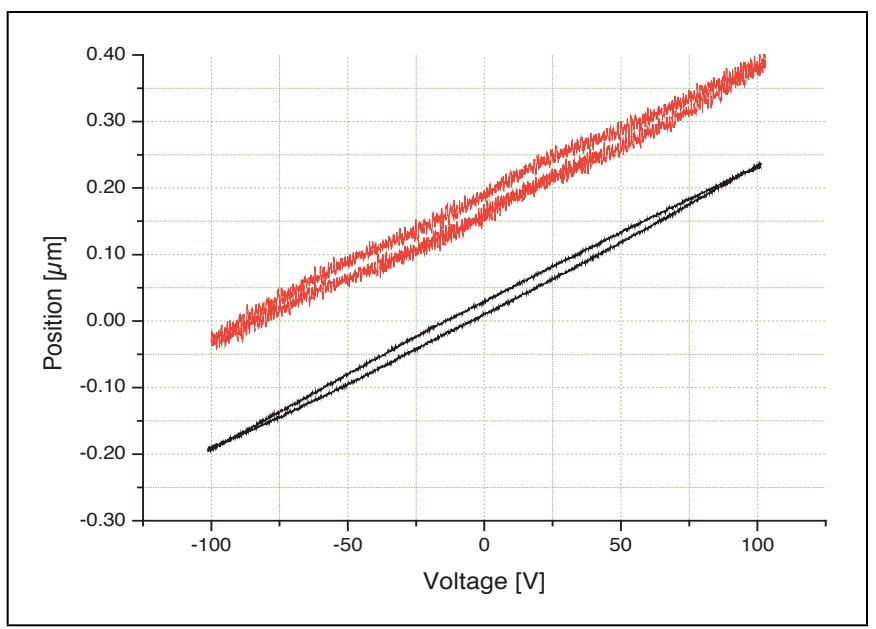

Fig. 2. Measurement on a transversal actuator. The upper curve represents the optical sensor output (shifted for better visibility), the lower curve the resistance measurement. The TFR output was scaled to match the amplitude of the optical sensor signal.

They exhibit a rather strong piezoresistive effect [6], which is thought to be due to strain inhomogeneity in their granular structure [7] and makes them useful for mechanical (force, pressure) sensor applications [8]. Thick-film based piezoresistive bridges can be applied using screen printing, which allows excellent integration and low-cost volume fabrication. For their application onto PZT substrates, a few important points are outlined below.

- In order to avoid capacitive coupling with the PZT, the bridge circuit should be shielded by sandwitching a ground plane between it and the PZT.

- The thick-film electrodes should adhere well and be chemically compatible with the PZT.

- As firing of thick-films involves high temperatures (typ. $850^{\circ} \mathrm{C}$ ), the actuators must be poled after fabrication of the piezoresistive bridge.

- Thick-film dielectrics and resistors are brittle, glassy materials whose strain limit is not known precisely. Application on alumina to ca. 500 ppm is known to be safe [9].

\section{PIEZO POSITIONERS WITH INTEGRATED TFR STRAIN GAUGE SENSORS}

\section{A. Transversal actuators}

Transversal actuators use the displacement perpendicular to the poling direction of the piezoelectric element (Figure 1). As the displacement is proportional to the length of the piezoceramic element, strokes of several $100 \mathrm{~nm}$ or even in the micron range 
can easily be obtained even with elements consisting of only one layer of piezolelectric material.

The displacement $\delta$ of a transversal actuator having the thickness $t$, the length $l$ with the applied Voltage $V$ and without external forces (free strain) is

$$
\delta=d_{31} l \frac{V}{t}
$$

The first experiments using TFR's as position feedback have been performed on these actuators to verify the functionality of the combination of PZT with TFR sensors.

The actuator material was a commercially available, $1 \mathrm{~mm}$ thick PZT sheet with $d_{31}=-210$. $10^{-12} \mathrm{~m} / \mathrm{V}$. Thick-film Ag:Pd:Pt (4\% Pd+Pt) conductor material was used for electrode pattern. A glassceramic dielectric layer was then applied in order to insulate the sensing bridge from the PZT drive electrodes. The resistor itself was made of a thickfilm resistor composition $(\mathrm{R} \square=10 \mathrm{k} \Omega$ ) with $\mathrm{Ag}: \mathrm{Pd}$ 3:1 terminations. The typical thickness for resistors, electrodes/terminations, and dielectric were $15 \mu \mathrm{m}$, $12 \mu \mathrm{m}$, and $40 \mu \mathrm{m}$ respectively. Actuators with a width of $3 \mathrm{~mm}$ and a free length of about $14 \mathrm{~mm}$ were fabricated. After firing the thick-film layers the material has been re-poled at $2 \mathrm{kV} / \mathrm{mm}$.

Comparative measurements have been performed using an optical sensor (triangulation type, $10 \mathrm{~nm}$ resolution) and the strain gauge output in a Wheatstone bridge setup. As the noise level of the optical sensor is large compared to the actuator displacement, a 32 fold averaging was used on the signal obtained from the TFR on the actuator driven with a $10 \mathrm{~Hz}$ triangle wave at an amplitude of $100 \mathrm{~V}$. The result (Figure 2) shows that sensor output and TFR measurement agree well.

It is important to notice that because of the asymmetry of the layers on upper and lower side of the actuator, a bending deformation is superposed to the desired transversal movement. In our particular case it was measured to be between $0.6 \mu \mathrm{m}$ and $1 \mu \mathrm{m}$ for a free actuator length of $14 \mathrm{~mm}$. Although this actuator might not be useful for its displacement in transversal direction, the resulting bending effect might be considered to produce inexpensive monomorph actuators.

\section{B. Bimorph actuators}

Bimorph elements [1] consist of at least two active piezoceramic layers. A different extension or con-

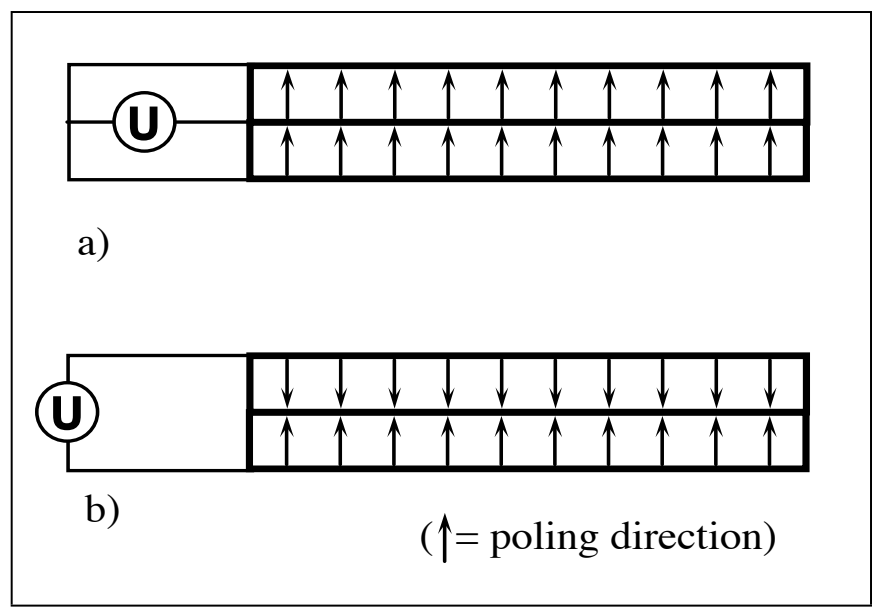

Fig. 3. Poling of bimorph actuators, a: parallel actuator type, b: serial actuator type

traction of one or more layers is caused by applying different electric fields on the layers. The ceramics are usually co-fired, which means that the layered structure and all necessary inner electrodes are made in one sintering process. The two layers are poled either in the same or in opposite directions (Figure 3). Actuators which have their layers poled in opposite directions (serial type) are electrically operated in series and require thus just two connections. Bimorph with the same poling direction for both layers have the advantage that the outer electrode may be used as ground, and the poling procedure is technologically easier to realize.

The displacement $\delta$ of a bimorph can be calculated as follows (note that $\mathrm{V}$ is the Voltage applied to one of the two layers):

$$
\delta=\frac{3 d_{31} l^{2} V}{t^{2}}
$$

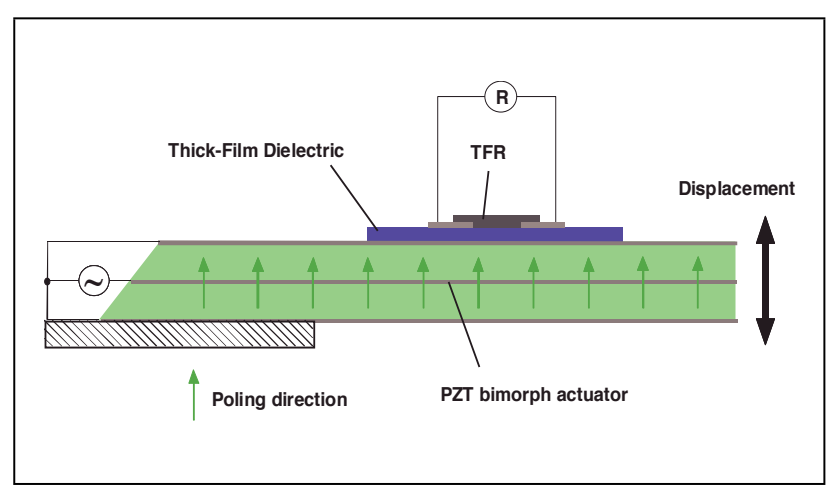

Fig. 4. Principle of TFR sensor integration on a bimorph

Figure 4 shows an example for the integration of a thick-film resistor directly on a co-fired bimorph. 
For applications using a Wheatstone bridge a second resistor can be integrated on the same side of the bimorph ceramic sheet or the full bridge may be integrated by placing two resistors on the top and two at the bottom of the sheet.

Monomorphs (also called unimorphs or heterogeneous bimorphs) are bonded using epoxy adhesives. In certain cases, such as if using a metal shim between the PZT ceramic sheets, bimorphs are also bonded using epoxies.

Laboratory tests have been conducted on samples made for these experiments with a commercial PZT material with $d_{31}=-160 \cdot 10^{-12} \mathrm{~m} / \mathrm{V}$. The layer composition for the TFR was the same as for the transversal actuators. Beams with about $40 \mathrm{~mm}$ free length, $5 \mathrm{~mm}$ width and $0.5 \mathrm{~mm}$ thickness per layer have been fabricated.

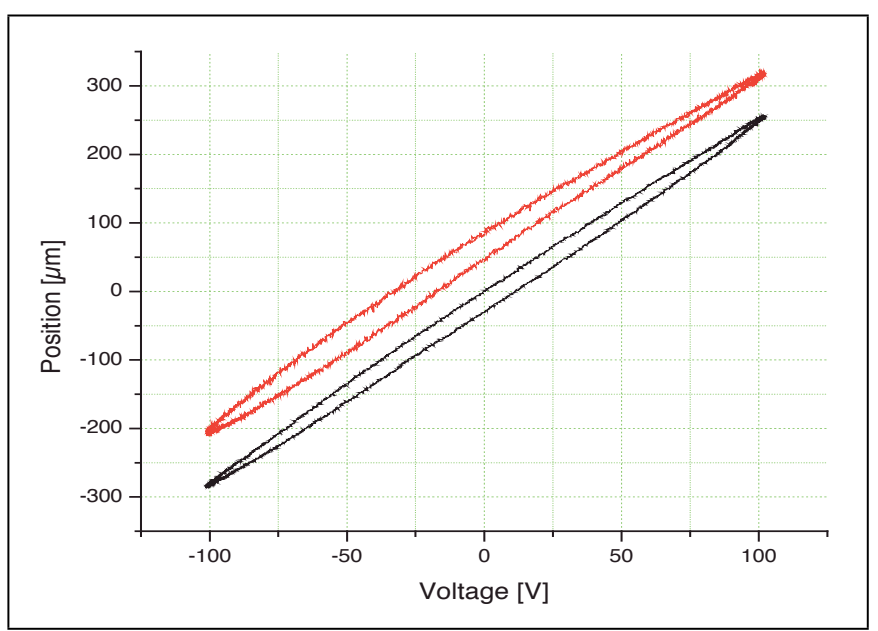

Fig. 5. Measurement on a bimorph actuator. The upper curve represents the optical sensor output (shifted for better visibility), the lower curve the resistance measurement. As in figure 4, the TFR output was scaled to match the amplitude of the optical sensor signal.

The comparison of the optical sensor output shows again a good agreement with the output from the bridge circuit with the TFR 5 . The theoretical deflection for a beam with $40 \mathrm{~mm}$ length of the abovementioned material at $100 \mathrm{~V}$ is $307 \mu \mathrm{m}$, the measured value is about $300 \mu \mathrm{m}$.

\section{C. planar bimorph}

The planar bimorph actuator (Figure 6) has certain advantages over other bimorph or monomorph types. It is very simple to fabricate; a bulk ceramic sheet is patterned with at least two different electrodes on one side and at least one electrode on the opposite side. Applying opposite voltage patterns yields a horizontal deformation. Yao et al. [10] even proposed and

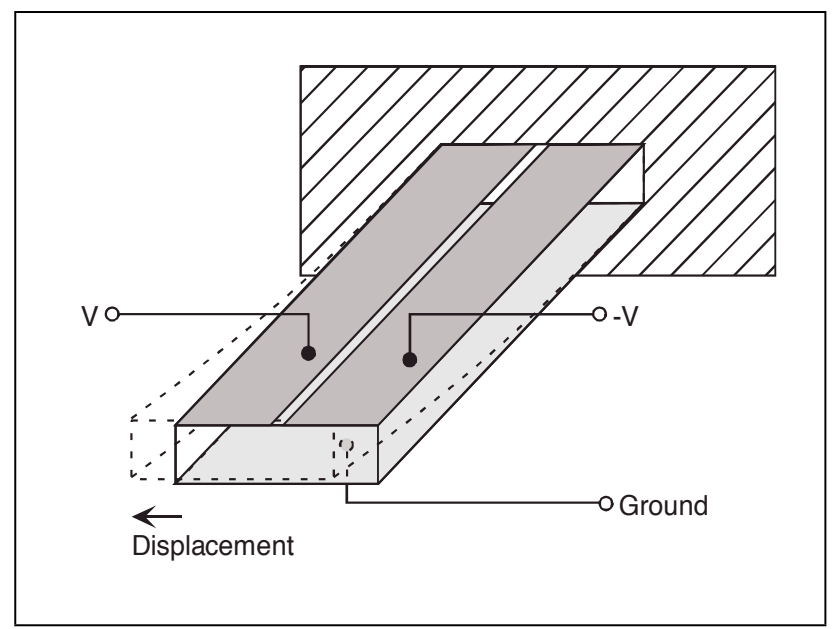

Fig. 6. Working principle of a planar bimorph actuator

analyzed a multilayered structure for this type of actuator.

The planar bimorph actuator is particularly interesting for the integration of TFR's as sensors. The actuator structure is defined by the electrode patterns, and a whole Wheatstone bridge can be patterned on one side of the bulk material.

According to [10] the displacement $\delta$ at the end of the beam is

$$
\delta=\frac{3 l^{2}\left(w^{2}-w_{0}^{2}\right)}{2 w^{3} t} d_{31} V
$$

with $l$ being the length of the electrode, $w$ the width of the whole beam, $t$ its thickness and $w_{0}$ the width of the gap between the two electrodes on both sides.

Measurements have been performed with beams as depicted in Figure 8, which were made of commercially available piezoceramic sheets with $d_{31}=$ $-210 \cdot 10^{-12} \mathrm{~m} / \mathrm{V}$. The resistors have been patterned with a particular shape in order to measure the strain of the actuator as close as possible to its edge without degrading the TFR or the contact between the TFR and the conductive tracks while cutting.

The PZT sheet has been patterned with a ground electrode covering the whole actuator on one side, and with a structured electrode on the other side, which results in the desired bimorph function. In the following step, a dielectric has been deposited, followed by the conductive layer for the connecting tracks and the resistive layer. After firing the deposited layers the ceramic sheets have been poled at $2 \mathrm{kV} / \mathrm{mm}$ and cut using a dicing saw as used in MEMs fabrication. 
The theoretical deflection of such a planar bimorph at an operating Voltage of 100 Volt calculated according to equation 5 and according to FEM simulations is about $7.4 \mu \mathrm{m}$ for an active length of $29 \mathrm{~mm}$.

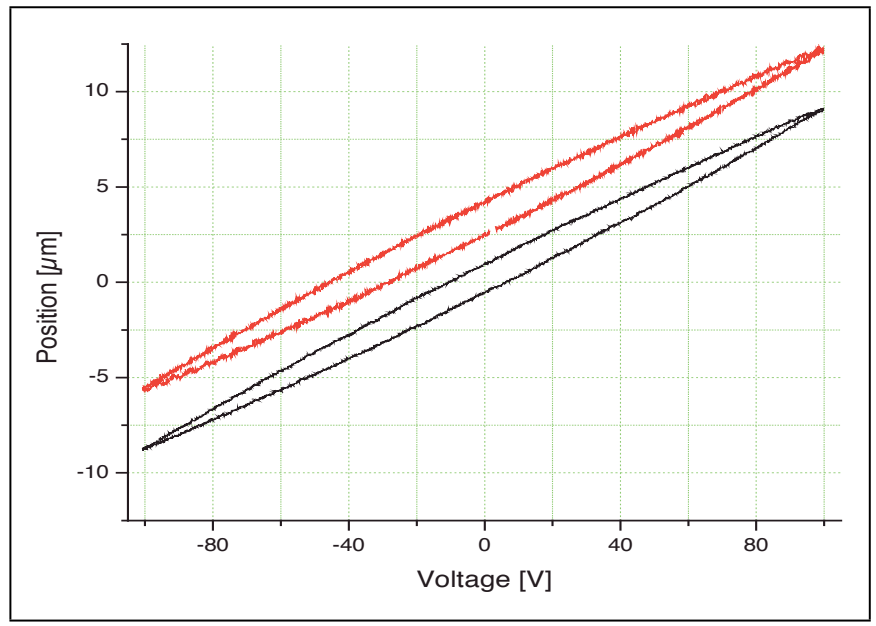

Fig. 7. Comparison of the bridge output with the position measurement for the planar bimorph actuator with integrated full Wheatstone bridge

Comparative measurements have been performed between the bridge output and the position, which was measured with a triangulation sensor as in the previous experiments and the resistances. As a complete bridge was integrated we used a common instrumentation amplifier (INA 114) to measure the bridge output directly. Figure 7 shows the resulting curves from the optical sensor and scaled bridge output.

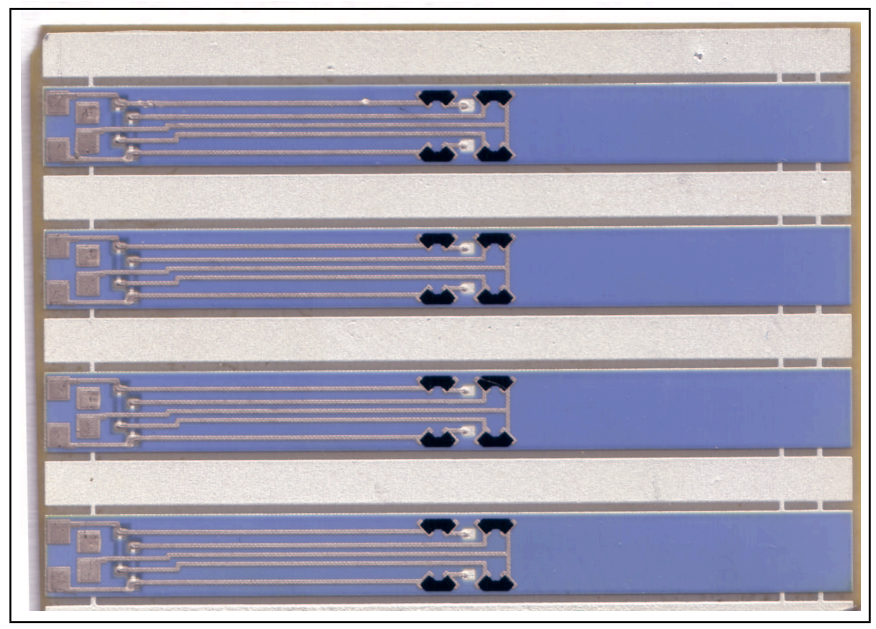

Fig. 8. PZT sheet with four actuators before dicing. A whole Wheatstone bridge is directly patterned onto each beam

For certain applications a particular problem with this type of actuator needs to be considered. As the bimorph bends horizontally, perpendicular to the

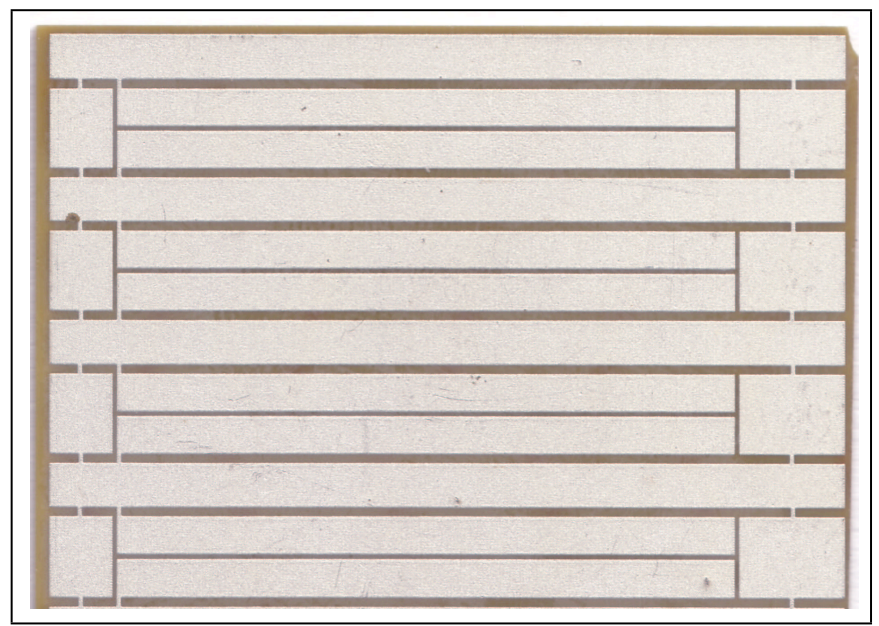

Fig. 9. backside of the structured PZT sheet

planes where the additional structures have been applied, there is a parasitic movement out of the horizontal plane if the layers on top and bottom side do not have the same elasticity and thickness, similar to the transversal actuator mentioned earlier.

This displacement will be sufficiently small to be neglected in certain applications, otherwise countermeasures have to be taken to suppress such a movement. Layers of the same thickness and with the same mechanical properties may be applied to the opposite side, or a design of the positioning system which is tolerant to this deflection may be considered.

In our setup this deflection was in the order of $4.5 \mu \mathrm{m}$ for an applied voltage of $100 \mathrm{~V}$, which represents about $20 \%$ of the actuator amplitude.

\section{ApPLICATIONS}

The proposed TFR sensors offer advantages wherever a very small sensor needs to be integrated into a piezoelectric positioning system. If technological and design problems are mastered, a multitude of applications in microrobotics and microhandling can be found.

One possible application would be in positioning systems for microscopy. Two bimorph beams could be combined to form an $x-y$ stage. With ranges of (depending of the dimensions) several $10 \mu \mathrm{m}$ this is potentially a solution occupying a very small space (e.g. $50 \mathrm{~mm} \times 50 \mathrm{~mm} \times 2 \mathrm{~mm}$ with additional mecanical elements such as flexure hinges etc.) at a very low cost for small assembly, measurement or positioning applications. 
Another possible application could be in microand nanotesting of materials (scratching and indentation tests). The properties of very small zones in the order of several $\mu \mathrm{m}^{2}$ are interesting here, and position and force need to be measured precisely. Integration of TFT's on PZT's would allow the conception of very small and inexpensive microtesting systems.

Although tests have not been performed yet it is supposed that the proposed technology is applicable to multilayer actuators as well, which opens an even wider application field.

\section{CONCLUSION AND OUTLOOK}

A new inexpensive integrated sensor for piezoelectric elements based on thick-film resistors has been presented. It has been shown that in comparison to relatively expensive state-of-the-art optical sensing systems, such as triangulation sensors, the performance is comparable or even better because of the lower noise level, while the sensors offer all advantages of TFR based force- and position-sensing systems (size, cost, robustness etc.)

This technology can potentially be used to replace strain gauges as well as other sensors in many applications where the deformation of a piezoelectric actuator needs to be measured.

Several problems have to be treated in the future to render this technology usable for applications:

- The parasitic deflection which occurs, when the TFR is not mounted perpendicularly to the bending plane (as on the transversal or planar bimorph actuators) needs to be compensated. This deflection, on the other hand, might be useful to create inexpensive monomorph actuators.

- Especially the planar bimorph actuator has shown, that a calibration of the bridge is indispensable if the whole bridge is integrated. For example, one or more additional TFR's at nonactive zones of the PZT could be integrated for laser trimming.

- The design (position and shape of the resistors needs to be optimized. It would furthermore be advantageous to integrate the pre-amplifier directly on the PZT plate on inactive zones.

- We suppose that the application to multilayer systems is possible to a certain extent. The respective technology needs to be developed and actuators have to be characterized.
- Experiments have been performed at low frequencies (quasi-static). It is important to know how well the TFR's can be used for dynamic applications.

- It is important to know about eventual ageing problems of the combination PZT/TFR. Experiments have been carried out during several hours in the laboratory without any observable degradation. Longer testing durations will be needed if reliable systems for commercial use are to be developed using TFR's.

\section{Acknowledgements}

The authors would like to thank Dr. T. Morita (EPFL-LC) for his help on the bimorph actuators, as well as C. Jacq and M. Garcin (EPFL-LPM) for the layout and fabrication of the planar bimorph actuators.

This work was partially supported by the Swiss National Science Foundation, project Nr. 2100-055404.98/1.

\section{REFERENCES}

[1] K. Uchino, Piezoelectric Actuators and Ultrasonic Motors. Boston, Dordrecht, London: Kluwer Academic Publishers, 1997.

[2] Physik Instrumente (PI) GmbH \& Co. KG, "http://www.physikinstrumente.com/." Web Site, 2002.

[3] Piezosystem Jena GmbH, "http://www.piezojena.de/." Web Site, 2002.

[4] J. Gardner, Microsensors - Principles and Applications. Chichester, New York, Brisbane, Toronto, Singapore: John Wiley \& Sons, 1994.

[5] M. Prudenziati and R. Dell'Acqua, Thick Film Resistors, vol. 1 of Handbook of Sensors and Actuators, pp. 85-97. Amsterdam: Elsevier Science, 1994.

[6] B. Morten and M. Prudenziati, Piezoresistive thick-film sensors, vol. 1 of Handbook of Sensors and Actuators, pp. 189-208. Amsterdam: Elsevier Science, 1994.

[7] C. Grimaldi, P. Ryser, and S. Straessler, "Gauge factor enhancement driven by heterogeneity in thick-film resistors," Journal of Applied Physics, vol. 90, no. 1, pp. 322-327, 2001.

[8] N. M. White and J. D. Turner, "Thick-film sensors: Past, present and future," Measurement Science and Technology, vol. 8, no. 1, pp. 1-20, 1997.

[9] D. Crescini, D. Marioli, and A. Taroni, "Piezoresistive accelerometers for vehicle dynamics: A new solution in thick-film technology on AISI430 metal substrate," Sensors and Materials, vol. 8, no. 7, pp. 431-438, 1996.

[10] K. Yao, W. Zhu, K. Uchino, Z. Zhang, and L. C. Lim, "Design and fabrication of a high performance multilayer piezoelectric actuator with bending deformation," IEEE Transactions on Ultrasonics, Ferroelectrics, and Frequency Control, vol. 46, no. 4, pp. 1020-1027, 1999. 